STRANGER FICTIONS 



\section{STRANGER FICTIONS}

A HISTORY OF THE NOVEL

IN ARABIC TRANSLATION

REBECCA C. JOHNSON 
Publication of this book was made possible, in part, by a grant from the Alice Kaplan Institute for the Humanities, Northwestern University.

\section{Copyright (C) 2020 by Cornell University}

All rights reserved. Except for brief quotations in a review, this book, or parts thereof, must not be reproduced in any form without permission in writing from the publisher. For information, address Cornell University Press, Sage House, 512 East State Street, Ithaca, New York 14850. Visit our website at cornellpress.cornell.edu.

First published 2020 by Cornell University Press

Library of Congress Cataloging-in-Publication Data

Names: Johnson, Rebecca C. (Rebecca Carol), author. Title: Stranger fictions : a history of the novel in Arabic translation / Rebecca C. Johnson.

Description: Ithaca [New York] : Cornell University Press, 2020. Includes bibliographical references and index.

Identifiers: LCCN 2020013041 (print) | LCCN 2020013042 (ebook) | ISBN 9781501753060 (hardcover) | ISBN 9781501753077 (ebook) | ISBN 9781501753305 (pdf)

Subjects: LCSH: Arabic fiction-European influences. | Arabic fiction-1801-History and criticism. | Translating and interpreting.

Classification: LCC PJ7577 .J64 2020 (print) | LCC PJ7577 (ebook) | DDC 892.7/35094—dc23

LC record available at https://lccn.loc.gov/ 2020013041

LC ebook record available at https:// lccn.loc.gov / 2020013042

Cover illustration: Samir Sayegh, Alef, 2014. Acrylic on wood pane, $60 \times 60 \times 2.5 \mathrm{~cm}$. Image courtesy of Barjeel Art Foundation, photographed by Capital D. 
For Nadim 
